\title{
Genetic testing for lymphedema in RASopathies
}

\author{
Stefano Paolacci ${ }^{1}$, Yeltay Rakhmanov ${ }^{2}$, Paolo Enrico Maltese ${ }^{2 \star}$, Alice Bruson ${ }^{2}$ and Matteo Bertelli ${ }^{1,2}$
}

\begin{abstract}
Variants affecting the function of genes in the RAS-mitogen-activated protein kinase (MAPK) signal transduction pathway have been identified as responsible for a group of developmental syndromes known as RASopathies. Noonan (NS) and cardiofaciocutaneous syndromes (CFC) represent the most frequent and best characterized RASopathies. Many cases of RASopathies are associated with lymphatic malformations that finally may result in lymphedema. We developed the test protocol "Lymphedema in RASopathies" on the basis of the latest research findings and diagnostic protocols on lymphatic malformation in RASopathies. The genetic test is useful for confirming diagnosis, as well as for differential diagnosis, couple risk assessment and access to clinical trials.
\end{abstract}

Keywords: Primary lymphatic malformations, Noonan syndrome, Noonan-like syndrome, RASopathies, EBTNA UTILITY GENE TEST

'MAGI Euregio, Bolzano, Italy

${ }^{2}$ MAGI's Lab, Rovereto, Italy

*Corresponding author: P. E. Maltese E-mail: paolo.maltese@assomagi.org

DOI: 10.2478/ebtj-2018-0025
(C) 2018 Authors. This work was licensed under the Creative Commons AttributionNonCommercial-NoDerivs 3.0 License.

\section{RASopathies}

(Other synonyms: Disorders related to the Ras/MAPK pathway, Noonan spectrum disorders, Noonan-like syndrome)

\section{General information about the disease}

Variants affecting the function of genes in the RAS-MAPK signal transduction pathway have been identified as responsible for RASopathies (1). The most common and studied RASopathies, Noonan syndrome (NS) and cardiofaciocutaneous syndrome (CFC), are characterized by multiple congenital anomalies (MCA). More than 50 types of congenital anomalies have been described in both syndromes (2). The cutaneous manifestations may include transient lymphedema of the dorsum of the hands and feet in infancy and stationary or progressive lymphangectatic edema in adulthood (3).

Prevalence of NS is about 1 in 1000 and 1 in 2500 live births $(4,5)$, whereas the prevalence for CFC is unknown.

The literature describes variable lymphatic abnormalities associated with NS and CFC. For example, a cross-sectional cohort study of 35 NS patients found a $49 \%$ prevalence of lymphedema, which was chronic and intermittent in nature and occurred at the extremities (6).

To date, 17 genes have been identified as associated with RASopathies: PTPN11, SOS1, RAF1, KRAS, HRAS, MAP2K1, MAP2K2, NRAS, CBL, SHOC2, BRAF, RIT1, A2ML1 (7), LZTR1, SOS2, SPRED1 and NF1. The disease arises from the hyperactivation of the RAS/ MAPK pathway, which regulates cell proliferation, differentiation and migration (6). This hyperactivation is caused by gain-of-function mutations in genes like PTPN11 that activate the signalling in response to growth factors (8), or loss-of-function mutations in genes that negatively regulate this pathway, such as NF1 (9). Over half of NS cases are caused 
by mutations in PTPN11, which encodes SHP2, a tyrosine phosphatase (10). SOS1 gene mutations cause an additional 10 to $15 \%$, and RAF1 and RIT1 genes each account for about $5 \%$ of cases (11). Mutations in other genes each account for a small number of cases. The molecular cause of NS in 15 to $20 \%$ of people with this disorder is unknown.

CFC is mainly caused by mutations in BRAF $(\sim 75 \%)$, followed by mutations in MAP2K1 or MAP2K2 ( 25\%), and KRAS ( 1\%) (11). Other genes are less frequently found mutated in CFC patients.

RASopathies are inherited in autosomal dominant pattern.

\section{The following phenotypic variants might manifest with lymphatic malformations:}

- Noonan syndrome 1 (NS1, OMIM disease 163950) PTPN11 (OMIM gene 176876);

- Noonan syndrome 3 (NS3, OMIM disease 609942) - KRAS (OMIM gene 190070);

- Noonan syndrome 4 (NS4, OMIM disease 610733) - SOS1 (OMIM gene182530);

- Noonan syndrome 6 (NS6, OMIM disease 613224) - NRAS (OMIM gene164790);

- Noonan syndrome 8 (NS8, OMIM disease 615355) - RIT1 (OMIM gene 609591);

- Noonan-like syndrome with or without juvenile myelomonocytic leukemia (NSLL, OMIM disease 613563) - CBL (OMIM gene 165360);

- Costello syndrome (CSTLO, OMIM disease 218040) - HRAS (OMIM gene 190020);

- Noonan-like syndrome with loose anagen hair (NSLH1, OMIM disease 607721) - SHOC2 (OMIM gene 602775);

- Cardiofaciocutaneous syndrome 1 (CFC1, OMIM disease 115150) - BRAF (OMIM gene 164757);

- Cardiofaciocutaneous syndrome 2 (CFC2, OMIM disease 615278) - KRAS (OMIM gene 190070);

- Cardiofaciocutaneous syndrome 3 (CFC3, OMIM disease 615279) - MAP2K1 (OMIM gene 176872);

Patients with RASopathies may show lymphatic anomalies due to the fact that RAS/MAPK pathway is strictly linked to other pathways associated with the onset of syndromes with the predominant involvement of the lymphatic system (12).

Pathogenic variants may include missense, nonsense, splicing, small insertions and deletions, small indels, gross deletions and insertions, and complex rearrangements.

\section{Aims of the test}

- To determine the gene defect responsible for the disease;

- To confirm clinical diagnosis;

- To assess the recurrence risk and perform genetic counselling for at-risk/affected individuals.

\section{Test characteristics}

Specialist centers/ Published Guidelines

The test is listed in the Orphanet database and is offered by 35 accredited medical genetic laboratories in the EU, and in the GTR database, offered by 21 accredited medical genetic laboratories in the US.

Guidelines for clinical use of the test are described in Genetics Home Reference (ghr.nlm.nih.gov) and Gene Reviews $(11,13)$.

\section{Test strategy}

Clinically distinguishable syndromes can be analyzed by sequencing only those genes known to be associated with that specific disease using Sanger or Next Generation Sequencing (NGS); if the results are negative, or more generally if clinical signs are ambiguous for diagnosis, a multi-gene NGS panel is used to detect nucleotide variations in coding exons and flanking introns of the above genes. Potentially causative variants and regions with low coverage are Sanger-sequenced. Sanger sequencing is also used for family segregation studies.

To perform molecular diagnosis, a single sample of biological material is normally sufficient. This may be $1 \mathrm{ml}$ peripheral blood in a sterile tube with $0.5 \mathrm{ml} \mathrm{K}$ EDTA or $1 \mathrm{ml}$ saliva in a sterile tube with $0.5 \mathrm{ml}$ ethanol $95 \%$. Sampling rarely has to be repeated. Gene-disease associations and the interpretation of genetic variants are rapidly developing fields. It is therefore possible that the genes mentioned in this note may change as new scientific data is acquired. It is also possible that genetic variants today defined as of "unknown or uncertain significance" may acquire clinical importance.

\section{Genetic test results \\ Positive}

Identification of pathogenic variants in the above genes confirms the clinical diagnosis and is an indication for family studies.

A pathogenic variant is known to be causative for a given genetic disorder based on previous reports, or predicted to be causative based on loss of protein function or expected significant damage to proteins or protein/protein interactions. In this way it is possible to obtain a molecular diagnosis in new/other subjects, establish the risk of recurrence in family members and plan preventive and/or therapeutic measures.

\section{Inconclusive}

Detection of a variant of unknown or uncertain significance (VUS): a new variation without any evident pathogenic significance or a known variation with insufficient evidence (or with conflicting evidence) to indicate it is likely benign or likely pathogenic for a given genetic disorder. In these cases, it is advisable to extend testing to the patient's relatives to assess variant segregation and clarify its contribution. In some cases, it could be necessary to perform further examinations/tests or to do a clinical reassessment of pathological signs.

\section{Negative}

The absence of variations in the genomic regions investigated does not exclude a clinical diagnosis but suggests the possibility of:

- alterations that cannot be identified by sequencing, such as large rearrangements that cause loss (deletion) or gain 
(duplication) of extended gene fragments;

- sequence variations in gene regions not investigated by this test, such as regulatory regions (5' and 3' UTR) and deep intronic regions;

- variations in other genes not investigated by the present test.

\section{Unexpected}

Unexpected results may emerge from the test, for example information regarding consanguinity, absence of family correlation or other genetically based diseases.

\section{Risk for progeny}

In autosomal dominant transmission, the probability that an affected carrier transmit the variant to his/her children is $50 \%$ in any pregnancy, irrespective of the sex of the child conceived.

\section{Limits of the test}

The test is limited by current scientific knowledge regarding the gene and disease.

\section{Analytical sensitivity (proportion of positive tests} when the genotype is truly present) and specificity (proportion of negative tests when the genotype is not present)

NGS Analytical sensitivity $>99.99 \%$, with a minimum coverage of 10X; Analytical specificity 99.99\%.

SANGER Analytical sensitivity $>99.99 \%$; Analytical specificity 99.99\%.

\section{Clinical sensitivity (proportion of positive tests} if the disease is present) and clinical specificity (proportion of negative tests if the disease is not present)

Clinical sensitivity is estimated at about $80-85 \%$ (13).

Clinical specificity: data not available.

\section{Prescription appropriateness}

The genetic test is appropriate when:

a) the patient meets the diagnostic criteria for Noonan syndrome or a RASopathy;

b) the sensitivity of the test is greater than or equal to that of tests described in the literature.

\section{Clinical utility}

\section{Clinical management}

Confirmation of clinical diagnosis

Differential diagnosis Yes

Couple risk assessment Yes

Availability of clinical trials can be checked on-line at https://clinicaltrials.gov/

\section{References}

1. Rauen KA. The RASopathies. Annu Rev Genomics Hum Genet 2013; 14: 355-69.

2. Kaplan MS, Optiz JM, Cosset FR. Noonan's syndrome: A case with elevated serum alkaline phosphatase levels and malignant schwannoma of the left forearm. Am J Dis Child 1968; 116(4): 35966.

3. Wyre HW Jr. Cutaneous manifestations of Noonan's syndrome. Arch Dermatol 1978; 114(6): 929-30.

4. Allanson JE. Noonan syndrome. J Med Genet 1987;24(1):9-13.

5. Sharland M, Burch M, McKenna WM, Patton MA. A clinical study of Noonan syndrome. Arch Dis Child 1992; 67(2):178-183.

6. Smpokou P, Tworog-Dube E, Kucherlapati RS, Roberts AE. Medical complications, clinical findings and educational outcomes in adults with Noonan syndrome. Am J Med Genet A 2012; 158A(12): 3106-3111.

7. Joyce S, Gordon K, Brice G, Ostergaard P, Nagaraja R, Short J, Moore $S$, Mortimer P, Mansour $S$. The lymphatic phenotype in Noonan and Cardiofaciocutaneous syndrome. Eur J Hum Genet. 2016; 24(5): 690-6.

8. Tartaglia M, Niemeyer CM, Fragale A, Song X, Buechner J, Jung A, Hählen K, Hasle H, Licht JD, Gelb BD. Somatic mutations in PTPN11 in juvenile myelomonocytic leukemia, myelodysplastic syndromes and acute myeloid leukemia. Nat Genet 2003; 34(2): 148-50.

9. Stark M, Assum G, Krone W. A small deletion and an adjacent base exchange in a potential stem-loop region of the neurofibromatosis 1 gene. Hum Genet 1991; 87(6): 685-87.

10. Tartaglia M, Kalidas K, Shaw A, Song X, Musat DL, van der Burgt I, Brunner HG, Bertola DR, Crosby A, lon A, Kucherlapati RS, Jeffery S, Patton MA, Gelb BD. PTPN11 mutations in Noonan syndrome: molecular spectrum, genotype-phenotype correlation, and phenotypic heterogeneity. Am J Hum Genet 2002; 70(6): 1555-63.

11. Rauen KA. Cardiofaciocutaneous syndrome. In: RA Pagon, MP Adam, HH Ardinger, SE Wallace, A Amemiya, LJH Bean, et al., editors. GeneReviews. 2016. Seattle (WA).

12. Brouillard $P$, Boon L, Vikkula M. Genetics of lymphatic anomalies. J Clin Invest 2014; 124(3): 898-904.

13. Allanson JE, Roberts AE. Noonan Syndrome. In: RA Pagon, MP Adam, HH Ardinger, SE Wallace, A Amemiya, LJH Bean, et al., editors. GeneReviews. 2001. Seattle (WA). 